\title{
The random allocation process: two things you need to know
}

Authors Joseph Dettori

Institution Spectrum Research, Inc. Tacoma, WA, USA

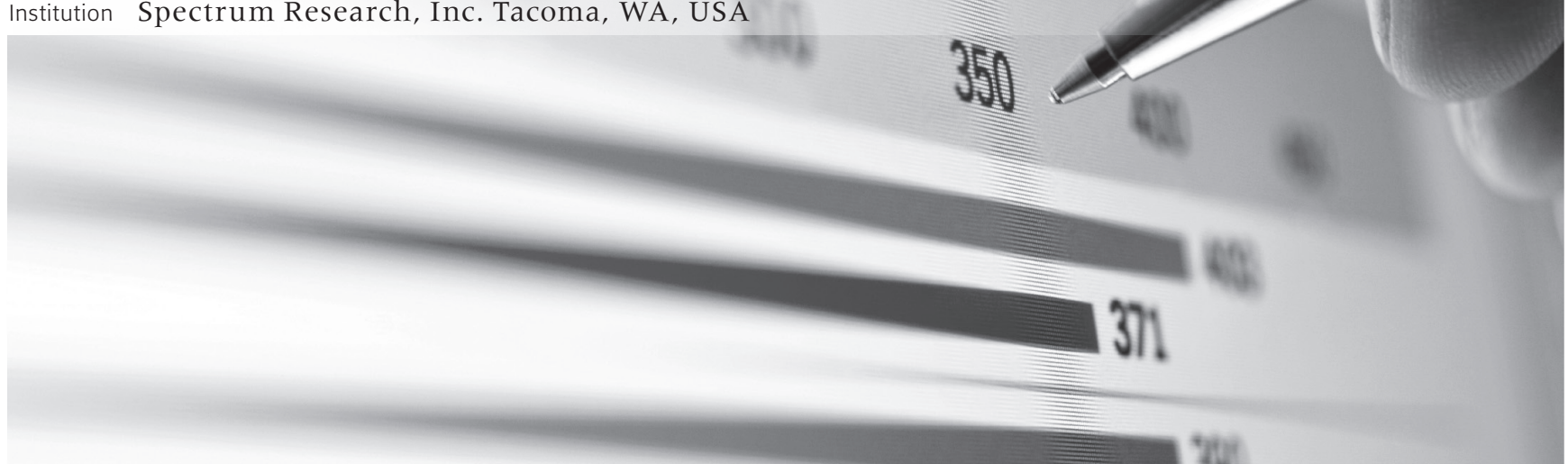

"The generation of random numbers is too important to be left to chance."-Robert $R$ Coveyou

The randomized controlled trial (RCT) has become the standard by which studies of therapy are judged. The key to the RCT lies in the random allocation process. When done correctly in a large enough sample, random allocation is an effective measure in reducing bias. In this article we describe the random allocation process.

\section{What makes up the random allocation process?}

The random allocation process consists of two steps:

1. generating an unpredictable random sequence,

2. implementing the sequence in a way that conceals the treatments until patients have been formally assigned to their groups.

\section{What are acceptable ways of generating a random sequence?}

Simple random allocation is the easiest and most basic approach that provides unpredictability of treatment assignment. In simple random allocation, treatment assignment is made by chance without regard to prior allocation (that is, it bears no relation to past allocations and it is not discoverable ahead of time).

Good methods of generating a random allocation sequence include using a randomnumbers table or a computer software program that generates the random sequence. There are manual methods of achieving random allocation such as tossing a coin, drawing lots or throwing dice. However, these manual methods in practice often become nonrandom, are difficult to implement and do not leave an audit trail. Therefore, they are not generally recommended. Procedures to avoid completely include using hospital chart numbers, alternating patients sequentially or assigning by date of birth.

Because simple random allocation has no relationship with prior assignment, unequal group sizes can happen by chance, especially in small sample sizes. To illustrate this point, 20 different random allocation sequences were generated for two 
treatments that had a total sample size of 20 patients. Here are the results of the number of patients randomly assigned to each of two treatment groups (A or B):

\begin{tabular}{lllllllllllllllllllllllll} 
& \#1 & $\# 2$ & $\# 3$ & $\# 4$ & $\# 5$ & $\# 6$ & $\# 7$ & $\# 8$ & $\# 9$ & $\# 10$ & $\# 11$ & $\# 12$ & $\# 13$ & $\# 14$ & $\# 15$ & $\# 16$ & $\# 17$ & $\# 18$ & $\# 19$ & $\# 20$ \\
\hline Group A & 13 & 10 & 10 & 12 & 4 & 9 & 9 & 12 & 13 & 11 & 10 & 12 & 13 & 8 & 10 & 11 & 10 & 7 & 6 & 6 \\
\hline Group B & 7 & 10 & 10 & 8 & 16 & 11 & 11 & 8 & 7 & 9 & 10 & 8 & 7 & 12 & 10 & 9 & 10 & 13 & 14 & 14 \\
\hline Difference & 6 & 0 & 0 & 4 & 12 & 2 & 2 & 4 & 6 & 2 & 0 & 4 & 6 & 4 & 0 & 2 & 0 & 6 & 8 & 8 \\
\hline
\end{tabular}

As you can see, an imbalance of six patients or more between groups occurred seven times (35\% of the time). In trial number five, the difference was twelve! However, this concern about group imbalance diminishes as the sample size gets bigger. In general for a two-arm trial, the probability of a significant imbalance is negligible with a sample size of 200 or more [1]. Alternatively, there are procedures other than simple random allocation that can be used to ensure balanced group sizes, such as blocking, the random allocation rule, and replacement randomization [1].

\section{What is allocation concealment?}

Allocation concealment is the technique of ensuring that implementation of the random allocation sequence occurs without knowledge of which patient will receive which treatment, as knowledge of the next assignment could influence whether a patient is included or excluded based on perceived prognosis.

For example, suppose that a spine surgeon has been working on a new kind of bone substitute that from a series of patients has shown great promise. The surgeon believes using this new substitute is better than the current method and wants to demonstrate this advantage in a randomized controlled trial. Let's also assume the random sequence has been generated, the new bone substitute is the next treatment to be given, and the surgeon knows that this treatment is next on the list. The next patient seen by the surgeon has comorbidities that make the surgeon believe that this patient is risky of achieving success with any treatment even though the patient meets the inclusion/exclusion criteria for the study. In this scenario one might easily subconsciously justify not enrolling the patient. Perhaps the patient hesitates briefly when the study is mentioned and the surgeon suggests that the patient sleep on the idea of participating. Maybe the surgeon decides to get more tests before offering enrollment. The number of different subtle possibilities to exclude this patient is only limited by one's imagination.

\section{What is the result when concealment is not ensured?}

One can expect a biased estimate of the treatment effect, and is in some cases as much as $40 \%$ or larger [2].

\section{What are acceptable ways to ensure concealment?}

The following are considered adequate approaches to concealed allocation:

- Central randomization. In this technique the individual recruiting the patient contacts a central methods center by phone or secure computer after the patient is enrolled.

- Sequentially numbered, opaque, sealed envelopes. This method is generally considered acceptable, but may be susceptible to manipulation [3]. If investigators use envelopes, it is suggested that the envelopes receive numbers in advance, and are opened sequentially, only after the participant's name is written on the appropriate envelope. In addition, the use of pressure sensitive paper inside the envelope should be used to transfer information to the assigned allocation. This can then serve as a valuable audit trail [4]. 


\section{Did the random allocation work?}

Researchers should always present the distributions of baseline characteristics by treatment group in a table (often the first table). This allows the reader to compare the groups at baseline on the distribution of important prognostic characteristics and allows surgeons to infer results to specific populations [5]. The reader should look for the magnitude of the differences between groups (if any are present) to see if those differences should be accounted for in the analysis.

The use of $P$-values to determine if differences in baseline characteristics are important is not appropriate in randomized trials $[4,6]$. Remember, the $P$-value is not a measure of the size of the effect, but is the probability that any differences are due to chance. In a trial with proper randomly generated and concealed allocation any differences at baseline are due to chance.

\section{Conclusions}

- The key phrase in an RCT is "random allocation" and it must be done properly, using two steps:

- generating the random sequence,

- implementing the sequence in a way that it is concealed.

- One should consider using a random numbers table or computer program to generate the random allocation sequence.

- To minimize the effect of bias, the random allocation sequence should remain concealed from those enrolling patients into the study.

\section{References}

1. Lachin JM (1988) Statistical properties of randomization in clinical trials. Control Clin Trials; 9(4):289-311.

2. Moher D, Pham B, Jones A, et al (1998) Does quality of reports of randomised trials affect estimates of intervention efficacy reported in meta-analyses? Lancet; 352(9128):609-613.

3. Bhandari M, Guyatt GH, Swiontkowski MF (2001) User's guide to the orthopaedic literature: how to use an article about a surgical therapy. J Bone Joint Surg Am; 83-A(6):916-926.

4. Schulz KF, Grimes DA (2002) Allocation concealment in randomised trials: defending against deciphering. Lancet; 359(9306):614-618.

5. Altman DG, Schulz KF, Moher D et al (2001) The revised CONSORT statement for reporting randomized trials: explanation and elaboration. Ann Intern Med; 134(8):663-694.

6. Altman DG, Doré CJ (1990) Randomisation and baseline comparisons in clinical trials. Lancet; 335(8682):149-153. 